\title{
Grupo de estudio y análisis de la morbi-mortalidad materna: Una ini- ciativa del sector privado para contribuir a la reducción en el número de muertes por esta causa en Bogotá, Colombia
}

\author{
Alberto Rizo G., MD, MSP*
}

\begin{abstract}
RESUMEN: Se ha establecido un Grupo de Estudio y análisis sobre la mortalidad materna en Bogotá, liderado por personas desde el sector privado que han ofrecido su colaboración al sector público para que se emprendan acciones encaminadas a reducir la incidencia de este fenómeno. El éxito, luego de dos reuniones celebradas en julio y en agosto de 1993, es relativo: no se ha logrado aún capitalizar el interés entre las autoridades del sector público que esta clase de esfuerzos supone. El entusiasmo de los impulsadores no obstante, se mantiene muy en alto y la decisión ha sido, continuar adelante hasta lograr que algún día se reconozca que una ciudad como la capital colombiana debe y puede alcanzar niveles de salud materna similares a las de cualquier capital del mundo industrial porque posee desde hace mucho tiempo los elementos suficientes para lograrlo.
\end{abstract}

SUMMARY: And ad hoc Study Group on the Analysys of the causes and consequences of Maternal Mortality has been organized by the private sector in Bogota, Colombia. The group serves as a forum were different topics affecting women's health such as maternal morbidity and mortality will be addressed. Participants from the Government, university professors from the Health Sciences institutions headquartered in Bogota, Women's organizations, several ONG's and the directors of the largest maternity hospitals in Bogota are invited to attend a series a 6-8 five hour meetings/year. It is intended that this group will watch the developments that take place in Colombia to secure that women's health will improve when the Government and the comunity work together towards achieving the set targets defined in the «Health for all in year 2000» strategy.

\section{Antecedentes}

La mortalidad materna en Colombia es uno de los indicadores de salud que no ha mostrado mejoría en los últimos 30 años pese al avance en el desarrollo social y económico que ha experimentado el país.

El Ministerio de Salud Pública estima que anualmente se producen alrededor de 2.000 defunciones en mujeres por causas relacionadas con el embarazo, el parto y el puerperio, cifra ésta que corresponde a una tasa anual de muertes maternas equivalente a 200 defunciones por cada 100.000 nacimientos. Si se observa el comportamiento de este indicador desde 1965 cuando alcanzaba cifras cercanas a 250 muertes por 100.000 , puede entenderse claramente que no se ha logrado mayor éxito en su reducción.

Colombia es país signatario de varias declaraciones desde Alma Ata en la ex Unión Soviética (1) tales como: La Conferencia sobre Maternidad sin Riesgos de Nairobi en 1987 (2), la Cumbre Mundial para la Infancia de 1991 (3) y la Conferencia Andina (4) sobre Maternidad sin Riesgos de comienzos de 1993 reunida en Santa Cruz, Bolivia, etc. En todos estos eventos los países firmantes han acordado emprender esfuerzos encaminados a disminuir drásticamente la morbi-mortalidad de ciertos grupos, especialmente el de madres y el de los niños con miras hacia la obtención de la meta «Salud para todos en el año 2000». En algunas áreas como en la de las enfermedades imunoprevenibles, la dia- rrea, la desnutrición infantil, la enfermedad aguda respiratoria, etc., Colombia ha logrado un indudable progreso.

En otras, existe un incumplimiento de los compromisos que ha firmado el país ya que las declaraciones que se formulan en los documentos oficiales (5) y la acción que se desarrolla en los distintos organismos que tienen a su cargo la organización y el desarrollo de los Programas de Salud, no muestran el esfuerzo que se requiere. Por ejemplo: la atención de la mujer durante el embarazo, el parto y el puerperio se hace en forma no muy diferente hoy si se compara con lo que se hacía en la década de los años sesenta, es decir, en forma rutinaria, sin lograr aún integrar redes de servicios eficientes que permitan el tránsito fluido de pacientes a los cuales se les ayude a resolver las situaciones que enfrentan, en fin, capaces de responder a las exigencias que requiere un programa moderno que de veras quiera romper de una vez por todas el círculo vicioso de causas que determinan las altas tasas de mortalidad materna vigentes en Colombia a finales del siglo XX.

Sucede sin embargo que el relativo bajo número de muertes maternas ( 2000 por año como ya se indicó) no parece preocupar a las autoridades, éstas sí muy alarmadas frente al número de muertes que producen los accidentes o los homicidios en casi todas las regiones de Colombia.

Este panorama motivó a un pequeño grupo de personas residentes en Bogotá a iniciar desde el sector privado, la 
creación de un grupo de estudio para contribuir con su aporte a reducir las altas cifras de mortalidad existentes en la capital como un primer paso hacia el logro de extender este esfuerzo a todo el país posteriormente.

\section{Situación actual de la mortalidad materna en Bogotá}

Basándose en estimados de población de hechos por el DANE (6) en los resultados de la encuesta de Demografía y Salud de 1990 (7) y en datos del Ministerio de Salud (8), el Comité elaboró para la ciudad de Bogotá las estimaciones que se aprecian en el cuadro siguiente (9):

ESTIMACIONES DE LOS NACIMIENTOS POSIBLES, ABORTOS Y DEFUNCIONES MATERNAS QUE OCURRIRAN EN BOGOTA PROYECCIONES PARA 1995 SEGUN DIFERENTES TASAS DE MORTALIDAD

\begin{tabular}{|c|c|c|c|c|c|c|c|}
\hline \multirow{2}{*}{$\begin{array}{l}\text { Nacimien- } \\
\text { tos espe- } \\
\text { rados } \\
(1)\end{array}$} & \multirow{2}{*}{$\begin{array}{l}\text { Abortos } \\
\text { (2) }\end{array}$} & \multicolumn{2}{|c|}{$\begin{array}{c}\text { Nacimientos } \\
\text { más } \\
\text { Abortos }\end{array}$} & \multicolumn{4}{|c|}{$\begin{array}{l}\text { Muertes maternas } \\
\text { esperadas según } \\
\text { diferentes tasas de } \\
\text { mortalidad por c/ } \\
100.000 \text { nacimientos }\end{array}$} \\
\hline & & (1)\&(2) & $\begin{array}{l}200 \\
(4) \\
\end{array}$ & $\begin{array}{l}175 \\
(5) \\
\end{array}$ & $\begin{array}{l}150 \\
(6) \\
\end{array}$ & $\begin{array}{l}50 \\
(7) \\
\end{array}$ & $\begin{array}{l}35 \\
(8) \\
\end{array}$ \\
\hline 120.000 & 21.176 & 141.176 & 282 & 247 & 211 & 62 & 49 \\
\hline 125.000 & 22.058 & 147.058 & 294 & 257 & 220 & 65 & 51 \\
\hline 130.000 & 22.941 & 152.941 & 305 & 267 & 229 & 67 & 53 \\
\hline 140.000 & 24.705 & 164.705 & 329 & 288 & 247 & 82 & 57 \\
\hline
\end{tabular}

Según el cuadro anterior, podrían presentarse en Bogotá hasta 329 muertes maternas anuales, de no haber cambios en las políticas de salud actuales, si se aplica la Tasa de Mortalidad por causas relacionadas con el embarazo, el parto y el puerperio estimada por el MSP para Colombia en 1993 (200 $x$ 100.000). En realidad, es posible que la Tasa de Mortalidad para Bogotá está probablemente ubicada entre 50 y 100 decesos por cada 100.000 nacimientos, con lo cual las defunciones maternas serían 110 a 120 cada año, cifra ésta que a pesar de ser menor que la que se calcula para el país, resulta inaceptablemente alta para una ciudad como la capital colombiana.

Ciudades como San Francisco, California en los EEUU o Santiago de Chile tienen tasas de defunciones maternas sensiblemente más bajas que las de Bogotá $(0.1$ y $2.5 \mathrm{x}$ 100.000 respectivamente) y estos niveles fueron alcanzados hace ya varios años (10-11).

Las causas de defunción durante el embarazo, el parto y el puerperio en la ciudad continúan siendo las mismas desde hace más de 25 años, es decir, hemorragia, infección, aborto, distocia y enfermedad hipertensiva del embarazo, con pequeñas variaciones entre éstas a lo largo del tiempo.

Puesto que las causas de defunciones maternas pueden enfrentarse con posibilidades de alcanzarse pleno éxito ya que no requieren intervenciones complejas ni tecnología muy sofisticada, es muy seguro que en Bogotá no se esté haciendo todo lo que es debido y de allí el interés del Grupo de Estudio y Análisis de la Mortalidad Materna para contribuir a que se conozcan las causas del problema y se pongan en práctica soluciones acordes con los recursos que posee la ciudad.
Estrategia propuesta: «Grupo de estudio y análisis de la Morbi-mortalidad materna en Bogotá» (12)

Profesionales de la salud, pertenecientes al sector privado que tuvieron la oportunidad de asistir a la Reunión de países del Grupo Andino celebrada en Santa Cruz, Bolivia, a comienzos de 1993, decidieron organizar una serie de reuniones con miras a la creación de un Grupo de Trabajo ad hoc, desde el sector privado. La sede provisional está ubicada en Oriéntame una ONG establecida desde hace varios años en Bogotá, la cual dedica su mayor esfuerzo a mejorar las condiciones de salud de una gran parte de mujeres residentes en la capital.

Entre los objetivos del grupo figuran constituir un foro para que se presenten iniciativas encaminadas a dar soluciones concretas para reducir la morbi-mortalidad materna tales como: creación de Comités de Vigilancia de la Mortalidad Materna en todos los hospitales de Bogotá; mejoramiento de los programas de prevención con amplia participación comunitaria; mejoramiento de la capacitación de los recursos humanos que prestan su concurso dentro de las instituciones privadas y gubernamentales de la capital; promoción para que la planificación familiar se amplíe a los sectores de la capital en los cuales la prevalencia de uso de anticonceptivos es aún baja; concientización a las autoridades de salud de Bogotá para que comprendan la importancia crítica que tienen el mejoramiento de los Bancos de Sangre, transporte y comunicaciones para atender la emergencia obstétrica; en un futuro proyecta canalizar solicitudes de investigaciones en el área de salud materna para buscar la cooperación de entidades nacionales y extranjeras que patrocinan estudios en esta área y en fin, liderar esfuerzos para la promoción hacia el logro de una ampliación total de la cobertura en la atención de la mujer durante el período prenatal, durante la atención del parto y en el postparto.

En todo momento se busca en las reuniones del grupo dar una amplia participación de entidades del sector oficial en los niveles nacional, departamental y distrital, las ONG's organizaciones feministas y entidades donantes como el Fondo de NNUU para Actividades de Población (FNUAP), la OPS, la AVSC y docentes de las escuelas de Ciencias de la Salud de Bogotá. Parte importante de los asistentes está conformada por los directores de todos los hospitales que atienden casos de maternidad en Bogotá. Un director, un coordinador y una secretaria, emplean un promedio de 10 horas (los dos primeros) y $20 \mathrm{hs}$., la última, en planear cada reunión del Grupo de Estudio y Análisis.

Se ha propuesto celebrar 3 reuniones durante 1993 y entre 6 y 8 en 1994 y años siguientes. Estos eventos tienen una duración de 5 horas en total (8:30 a 12:30 am), incluyen un descanso a media mañana y terminan con un almuerzo que se ofrece a todos los asistentes por cortesía de los organizadores. Las reuniones de amplia participación a los asistentes, se evalúan al final para recoger las opiniones sobre la organización, sobre las presentaciones efectuadas y sobre los aspectos tratados en el evento. Igualmente se recogen las sugerencias sobre temas para incluir en próximas reuniones.

$\mathrm{La}$ asistencia promedio en las dos primeras reuniones fue de 40 personas, con amplia participación de docentes universitarios, representantes de las entidades oficiales de salud de nivel nacional, departamental y distrital establecidas en Bogotá, representantes de agencias de cooperación internacional, etc. 
Los organizadores de este esfuerzo tienen en mente crear durante 1993 una masa crítica de personas que se interesen en el tema de la morbi-mortalidad materna en la capital colombiana. Durante 1994, este grupo debe consolidarse definitivamente y estar listo para presentar iniciativas a la nueva administración que se hará cargo de los destinos del país desde agosto de ese año. A partir de entonces, el grupo deberá continuar vigilando el desarrollo de los programas, las acciones legislativas que se emprendan y sirviendo de foro para promover temas que afecten la salud de la mujer, especialmente el de la morbi-mortalidad materna.

\section{Dificultades encontradas}

a) No es fácil en Colombia tratar de organizar esfuerzos como el que se propone el Grupo de Estudio y Análisis de la Mortalidad Materna de Bogotá. Pese a que se ha invitado a más de 60 personas a cada una de las reuniones, no se ha logrado atraer a los principales protagonistas, esto es, a quienes dirigen las principales maternidades de Bogotá, en las cuales muy seguramente se presenta el mayor número de defunciones maternas cada año. Lograr su importante participación es una labor que continuarán sin desmayo los organizadores de esta iniciativa. b) Las personas que acuden a las reuniones, no son por lo general las mismas, lo cual implica un esfuerzo grande de los organizadores para mantener informado a los asistentes sobre los propósitos del Grupo de Estudio y sobre los planes próximos y futuros del mismo.

c) El financiamiento de la organización de las reuniones (Correo, papelería, almuerzo, invitaciones, refrigerios, contactos con los ponentes de temas escogidos para presentar, alquiler del local, etc.) no se tiene asegurado completamente por cuanto no se ha logrado aún cristalizar el interés que de esfuerzos deberían mostrar entidades del sector oficial por iniciativas que le ayuden a tratar de resolver un problema de salud pública como el de la mortalidad materna. Baste citar que, además del sufrimiento humano que produce y el impacto negativo sobre la familia y sobre niños que quedan huérfanos, el número de años de vida productiva que se pierdan al morir una mujer durante cualquiera de las tres primeras décadas de la vida, representan varios miles de millones de pesos que se suman a los costos de otros lastres sociales y económicos que impiden el armónico desarrollo del país.

\section{BIBLIOGRAFIA}

1. WHO/UNICEF. Primary Health Care. International Conference on Primary Health Care. Alma Ata. USSR, 6-12 September 1978. Geneva: WHO, 1978.

2 . The International Safe Motherhood Conference. Nairobi, Kenya, 1987.

3. Cumbre Mundial para la Infancia. UNICEF, New York, 1991.

4. Conferencia Andina Maternidad sin Riesgos. Santa Cruz, Bolivia, marzo, abril 1993.

5. Convenio UNFPA- Ministerio de Salud, 1992, Bogotá, Colombia.

6. Proyecciones Nacionales de Población para Colombia 1950-2025 DANE, Bogotá, 1989.

7. Encuesta de Prevalencia, Demografía y Salud, 1990. Profamilia, DHS, Bogotá, 1991.
8. Ministerio de Salud, División de Salud Reproductiva: Plan de Acción para la Reducción de la Mortalidad Materna en Colombia, 1992. Bogotá, septiembre de 1992.

9. Rizo GA. Documentos en Mimeo preparatorios para I Reunión del Grupo de Estudio y Análisis de la Mortalidad Materna, Bogotá, julio 1993.

10. Viel B. y Campos W. Principales índices Biodemográficos y de Salud en Chile entre 1950 y 1987. Aprofa, Santiago, 1989.

11. Puffer R., Serrano C. Patterns of mortality in the Americas. PAHO, Washington, DC. 1975.

12. Villarreal J. y Rizo A. Grupo de Estudio y Análisis de la Mortalidad Materna. Objetivos. Mimeo, Bogotá, julio 1993. 